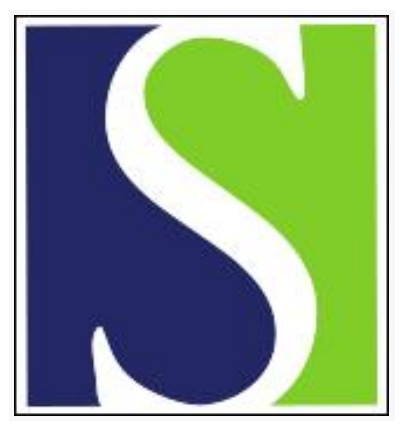

Scand J Work Environ Health 2008;34(3):169-178

https://doi.org/10.5271/sjweh. 1240

Issue date: 30 Jun 2008

Systematic review of interventions for reducing occupational stress in health care workers

by Ruotsalainen J, Serra C, Marine A, Verbeek J

Affiliation: Finnish Institute of Occupational Health, Cochrane Occupational Health Field, Neulaniementie 4, PO Box 93, FI-70701 Kuopio, Finland. jani.ruotsalainen@ttl.fi

Refers to the following text of the Journal: 2006;32(6):515-527

The following articles refer to this text: 2016;42(5):371-381;

2018;44(6):613-621

Key terms: anxiety; burnout; cognitive-behavioral therapy; health care worker; intervention; meta-analysis; nurse; occupational stress; review; systematic review

This article in PubMed: www.ncbi.nlm.nih.gov/pubmed/18728906 


\title{
Systematic review of interventions for reducing occupational stress in health care workers ${ }^{1}$
}

\author{
by Jani Ruotsalainen, MSc, ${ }^{2}$ Consol Serra, PhD, ${ }^{3}$ Albert Marine, MD, ${ }^{4}$ Jos Verbeek, PhD ${ }^{5}$
}

\begin{abstract}
Ruotsalainen JH, Serra C, Marine A, Verbeek JH. Systematic review of interventions for reducing occupational stress in health care workers. Scand J Work Environ Health. 2008;34(3):169-178.

Objectives This study evaluated the effectiveness of interventions in reducing stress at work among health care workers.

Methods A systematic search was conducted of the literature on reducing stress or burnout in health care workers. The quality of the studies found was then appraised and the results combined. A meta-analysis was performed when appropriate.

Results Altogether 14 randomized controlled trials, three cluster-randomized trials, and two crossover trials, comprising 2812 participants, were included. Only two trials were of high quality. The following comparisons were possible: person-directed interventions versus no intervention, person-work interface interventions versus no intervention, and organizational interventions versus no intervention. Person-directed interventions can reduce stress [standardized mean difference (SMD) - $0.85,95 \%$ confidence interval (95\% CI) - 1.21--0.49] and burnout, measured as emotional exhaustion [weighted mean difference (WMD) -5.82 , 95\% CI -11.02--0.63) and lack of personal accomplishment (WMD -3.61; 95\% CI -4.65--2.58). They also reduce anxiety, measured as state anxiety (WMD -9.42, 95\% CI -16.92--1.93) and trait anxiety (WMD -6.91, 95\% CI -12.80--1.01). Person-work interface interventions can reduce burnout, measured as depersonalization [mean difference (MD) $-1.14,95 \%$ CI -2.18--0.10]. Organizational interventions can also reduce stress symptoms (MD - 0.34 ; 95\% CI -0.62--0.06) and general symptoms (MD -2.90, 95\% CI -5.16--0.64). No harmful effects were reported. Conclusions Limited evidence is available for a small, but probably relevant reduction in stress levels from person-directed, person-work interface, and organizational interventions among health care workers. This finding should lead to a more-active stress management policy in health care institutions. Before large-scale implementation can be advised, larger and better quality trials are needed.
\end{abstract}

Key terms anxiety; burnout; cognitive-behavioral therapy; meta-analysis; nurse.

Changes in global economic realities are progressively transforming the very nature of work from physical tasks to more mental and emotional endeavors. The prevention of high stress levels in the work environment is thereby imperative in efforts to improve the quality of worklife, even in the face of increased job insecurity. Stress can be defined as a subjective psychophysiological state characterized by a combination of high arousal with displeasure. Although there is some controversy about the exact mechanism of work-related or occupational stress, the most extensively used models, demand-control (1) and effort-reward (2), explain it as an imbalance between particular factors. The resultant effects of stress on individual persons are mediated by personal factors like age, experience, health, coping skills, and the like (3-5).

Health care workers are no exception when it comes to suffering from work-related stress. Under some conditions, their work-related stress can lead to anxiety and depression, burnout, or psychosomatic diseases and

1 This paper was presented at the 28th International Congress on Occupational Health, 11-16 June 2006, in Milan Italy; at the 7th Conference of the European Academy of Occupational Health Psychology, 8-10 November 2006, in Dublin, Ireland; and at the XII Congres Sespas, 20-22 June 2007, in Barcelona Spain.

2 Finnish Institute of Occupational Health, Cochrane Occupational Health Field, Kuopio, Finland.

3 Unit of Occupational Health Research, University Pompeu Fabra, Barcelona, Spain.

4 Prevention Service, Corporacio Sanitaria Parc Tauli de Sabadell, Catalonia, Spain.

5 Cochrane Occupational Health Field, Coronel Institute of Occupational Health, Academic Medical Center, Amsterdam, Netherlands.

Correspondence to: Jani Ruotsalainen, Finnish Institute of Occupational Health, Cochrane Occupational Health Field, Neulaniementie 4, PO Box 93, FI-70701 Kuopio, Finland. [E-mail: jani.ruotsalainen@ttl.fi] 
a resultant deterioration in quality of life and service provision (6-9). Burnout has been defined as "a persistent, negative, work-related state of mind in 'normal' individuals that is primarily characterized by exhaustion, which is accompanied by distress, a sense of reduced effectiveness, decreased motivation, and the development of dysfunctional attitudes and behavior at work. This psychological condition develops gradually but may remain unnoticed for a long time for the individual involved. It results from a misfit between intentions and reality at the job. Often burnout is self-perpetuating because of inadequate coping strategies associated with the syndrome [p 388]" (4). Burnout is considered in this review as a form of psychological stress and not as a clinical diagnosis (10). The economic impact of such conditions is high, as can be inferred from data on absenteeism and turnover $(11,12)$.

There are many stress factors in the workplace of health care workers that have been shown to increase the risk of distress and burnout, for example, an increasing administrative workload, contact with suffering and dying patients, verbal and physical abuse by patients, bullying by colleagues, the need to hide negative emotional responses, risk of litigation, role conflicts between professions, and organizational changes (10, 13-15). In addition, many studies have shown that levels of dissatisfaction, distress, and burnout at work are high among health care workers $(12,16-20)$ and may even be higher than among workers in other occupations (7). There are numerous obstacles to conducting effective stress-related interventions at workplaces; therefore, it is all the more important to be systematic when determining what really works and what does not (21). Because health care workers form a relatively homogeneous and specific population, stress management interventions can be tailored to their specific needs, and consequently the results of a review concerning this occupational group may have a higher generalizability than synthesizing studies of various occupational groups. Therefore, we thought there was a need for a systematic review especially targeting health care workers.

Several reviews have been published on the effectiveness of interventions in preventing or treating stress (22-25). However, there is only one that has focused specifically on health care workers, and it did not reach clear conclusions about the evidence (26). The aim of this review was to ascertain the effectiveness of interventions in reducing stress in health care workers.

\section{Material and methods}

We performed a systematic literature search up to May 2005 to locate studies in electronic databases, including
MEDLINE, PsychINFO, the Cochrane Depression, Anxiety and Neurosis Group specialized registry, and the Cochrane Occupational Health Field database. References from articles and reviews were also reviewed, and all issues of Work \& Stress between January 1987 and May 2005 were hand-searched (27). [See the appendix for the MEDLINE search strategy that we employed.]

We included studies with interventions that were directed at workers who had not actively sought help for stress, burnout, depression, or anxiety disorder and in which interventions were compared with nonintervention controls or with alternative interventions. As outcomes, we considered all validated self-report measures of stress or burnout and all measures of the detrimental effects of stress or burnout.

Two reviewers independently checked each identified trial, determined inclusion, and graded the methodological quality with a previously validated checklist (28). Disagreements were resolved by consensus. We needed an instrument that could also assess the quality of the nonrandomized studies of organizational interventions that we wanted to include. The checklist's scales of internal validity were used for rating study quality, scores higher than $75 \%$ of the maximum of the two scales combined indicating high internal validity.

If interventions, participants, and outcomes were comparable, we pooled the results of individual studies. If sufficient numerical data were available, we performed a meta-analysis of outcomes by combining trials using the Mantel-Haenszel method (RevMan 4.2.8, The Nordic Cochrane Centre, Copenhagen, Denmark, 2003). Statistical heterogeneity was evaluated and $\mathrm{I}^{2}>50 \%$ was considered significant. Outcomes were summarized as standardized (SMD) or weighted (WMD) mean differences. A weighted mean difference can be calculated if all trials have measured the outcome on the same scale (eg, burnout with the Maslach Burnout Inventory). If the scales used differ but measure the same thing (eg, stress), then a standardized mean difference (MD) can be calculated. Individual trials affect both summary measures in proportion to their sample sizes so that the weight of a study is equal to the inverse of its variance. When there was significant heterogeneity, we applied a random-effects model; otherwise we used a fixed-effects model. For the remaining studies that did not report sufficient numerical data for pooling, we performed a qualitative synthesis (29). See table 1 for the system used to grade the evidence.

If a study used a cross-over design (two groups take turns being the intervention and the control group), we used the results from just after the first implementation of the intervention. One study (30) compared more than one active intervention; therefore, we entered the intervention that we judged to be the most intense into the meta-analysis to avoid having to input the same study more than once into the meta-analysis and thus 
Table 1. Qualitative synthesis of evidence [adapted from methods suggested by the Cochrane Back Review Group (29)].

\begin{tabular}{ll}
\hline Level of evidence & Contents \\
\hline Strong & $\begin{array}{l}\text { Two or more high-quality studies with similar positive or negative effects that are confirmed by the quantitative analysis } \\
\text { Limited }\end{array}$ \\
$\begin{array}{l}\text { Only one high-quality study or multiple low-quality studies or both, with similar positive or negative effects or the results of the } \\
\text { high-quality studies not confirmed by the quantitative analysis }\end{array}$ \\
$\begin{array}{ll}\text { Inconsistent } & \text { Results of studies point in opposite directions } \\
\text { No evidence } & \text { No studies available }\end{array}$ \\
\hline
\end{tabular}

compound its effect on the summary score. Using a fairly large assumed intracluster correlation of 0.10 , we calculated the design effect for cluster-randomized studies that had not considered it. Where necessary, missing statistics data were sought from authors. Since all of the studies used questionnaires as outcome measures, we were unable to conduct an intention-to-treat analysis. We considered the influence of publication bias, but we did not use funnel plots due to the small number of trials in each comparison group.

\section{Study characteristics}

Figure 1 shows the details of the exclusion and inclusion of studies. Table 2 shows the characteristics of the 19 included studies, of which 14 were randomized controlled trials, three were cluster-randomized trials, and two were cross-over studies. All three of the clusterrandomized trials (31-33) had a unit of analysis error; in other words, they analyzed the results at the individual level without taking the effect of the cluster design into account. The reasons for excluding nine studies from the meta-analyses were unique interventions like recreational music-making (34), therapeutic massage (35), support and advice from a psychologist $(31,36)$, and "primary nursing" (37), or insufficient reporting of outcome data $(33,38-41)$.

There was only one study (42) that reported using an acceptable method of randomization and concealing group allocation until it was completed and irrevocable. There was mention in only one study (43) of blinding those rating the outcome measurements. In all but one of the included studies (35), the blinding of the participants with respect to the intervention would have been impossible due to the nature and aims of the intervention being self-evident. The loss of participants was low throughout, and only two studies $(40,44)$ suffered a loss exceeding $20 \%$ of the initial sample. Two reviewers (JR and JV) independently rated all of the included studies on a quality checklist (28) (table 3). The Cohen's kappa of agreement was 0.62 for the internal validity scales. There were only two studies $(33,43)$ that were rated as being high in quality. Both of them were studies on organizational interventions.

Altogether 11 of the 19 included studies (30, 35, $37,38,42-48$ ) had interventions that were specifically

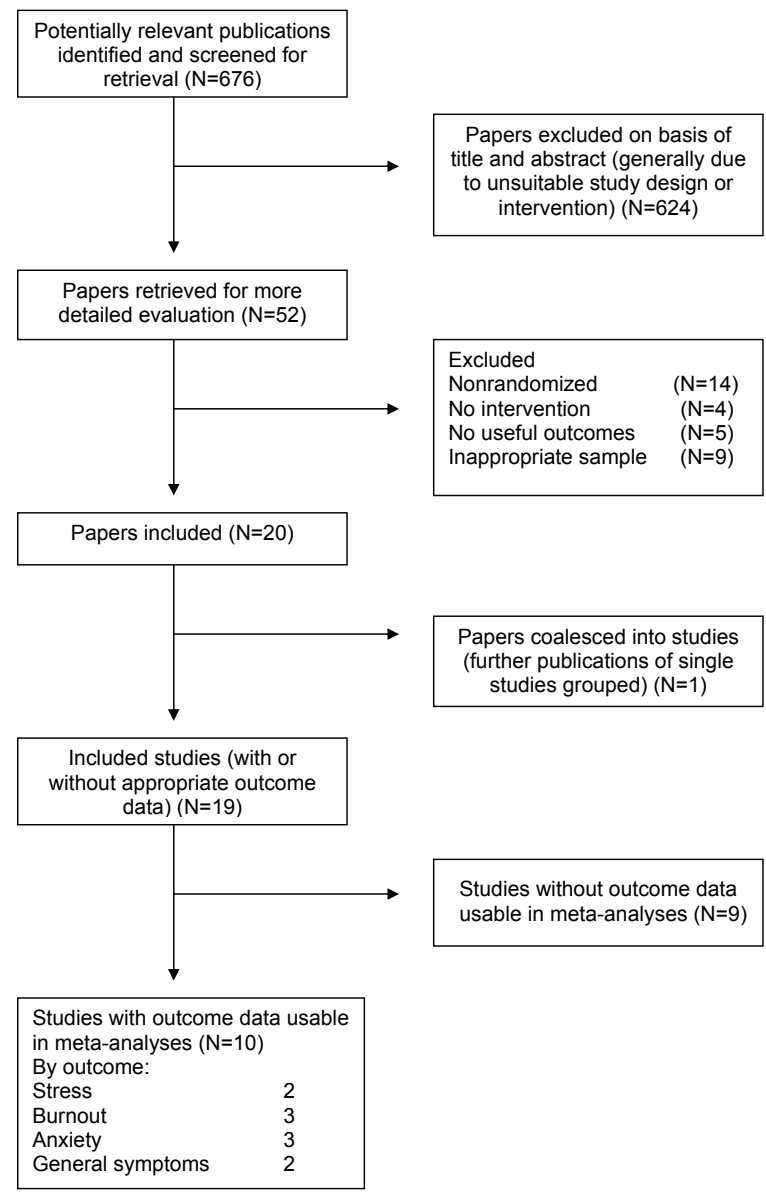

Figure 1. The inclusion and exclusion of trials.

directed towards nurses. In seven studies (32-34, 36, $39,49,50)$, interventions were directed at all of the staff of participating health care facilities, and, in one study, the intervention was directed towards respiratory therapists (40). We categorized interventions as (i) person-directed if they were aimed at changing personal characteristics without explicit reference to functioning at work, (ii) person-work interface intervention if they were aimed at improving the fit between the person and the organization (eg, role conflict-ambiguity, relationships, employee involvement in decision making), and (iii) organizational if they targeted organizational or social environments (eg, organizational restructuring, training, and job redesign) that may produce stress (22, 
Table 2. Characteristics of the included studies. The categorization of interventions was conducted using the typology of DeFrank \& Cooper (22). $(\mathrm{RCT}=$ randomized controlled trial, US = United States, UK = United Kindgom, STAI = State Trait Anxiety Inventory, SCL-90-R = Symptom Checklist-90-Revised, BDI = Beck Depression Inventory, DHEA = dehydroepiandrosterone)

\begin{tabular}{|c|c|c|c|c|c|}
\hline Study & Methods & Participants & Interventions (duration) & Outcomes & Target \\
\hline $\begin{array}{l}\text { Bittman et al, } \\
2003(34)\end{array}$ & $\begin{array}{l}\text { Cross- } \\
\text { over }\end{array}$ & $\begin{array}{l}112 \text { randomly selected } \\
\text { staff of a retirement com- } \\
\text { munity in the US }\end{array}$ & $\begin{array}{l}\text { Recreational music making; no intervention con- } \\
\text { trol (six 1-hour weekly sessions = } 6 \text { hours) }\end{array}$ & $\begin{array}{l}\text { Maslach Burnout Inventory, Profile } \\
\text { of Mood States }\end{array}$ & $\begin{array}{l}\text { Person-di- } \\
\text { rected }\end{array}$ \\
\hline $\begin{array}{l}\text { Cohen-Katz et al, } \\
2005(49)\end{array}$ & $\mathrm{RCT}$ & $\begin{array}{l}25 \text { nurses, pastoral care, } \\
\text { respiratory therapy and } \\
\text { social work personnel in } \\
\text { the US }\end{array}$ & $\begin{array}{l}\text { Mindfulness-based stress reduction program; } \\
\text { no intervention control (eight } 2.5 \text {-hour weekly } \\
\text { sessions and a 6-hour daylong retreat, } 8 \text { weeks } \\
=26 \text { hours) }\end{array}$ & $\begin{array}{l}\text { Maslach Burnout Inventory, brief } \\
\text { symptom inventory }\end{array}$ & $\begin{array}{l}\text { Person-di- } \\
\text { rected }\end{array}$ \\
\hline $\begin{array}{l}\text { Delvaux et al, } \\
2004(43)\end{array}$ & $\mathrm{RCT}$ & $\begin{array}{l}115 \text { oncology nurses in } \\
\text { Belgium }\end{array}$ & $\begin{array}{l}\text { Psychological training program; no interven- } \\
\text { tion control (three } 1 \text {-week courses, } 3 \text { months = } \\
105 \text { hours) }\end{array}$ & Nursing Stress Scale & $\begin{array}{l}\text { Organiza- } \\
\text { tional }\end{array}$ \\
\hline $\begin{array}{l}\text { Ewers et al, } 2002 \\
(38)\end{array}$ & RCT & $\begin{array}{l}20 \text { forensic mental health } \\
\text { nurses in the UK }\end{array}$ & $\begin{array}{l}\text { Psychosocial intervention training; no interven- } \\
\text { tion control ( } 20 \text { days } \sim 120 \text { hours) }\end{array}$ & Maslach Burnout Inventory & $\begin{array}{l}\text { Person-di- } \\
\text { rected }\end{array}$ \\
\hline $\begin{array}{l}\text { Heaney et al, } \\
1995(39)\end{array}$ & $\mathrm{RCT}$ & $\begin{array}{l}1375 \text { direct care staff and } \\
\text { home managers in the US }\end{array}$ & $\begin{array}{l}\text { Caregiver support program; no intervention con- } \\
\text { trol (six 5-hour sessions, } 9 \text { weeks = } 30 \text { hours) }\end{array}$ & $\begin{array}{l}\text { Social support, organizational } \\
\text { climate, SCL-90-R, confidence in } \\
\text { coping ability }\end{array}$ & $\begin{array}{l}\text { Person- } \\
\text { work } \\
\text { interface }\end{array}$ \\
\hline $\begin{array}{l}\text { Jones \& } \\
\text { Johnston, } 2000 \\
(42)\end{array}$ & $\mathrm{RCT}$ & $\begin{array}{l}79 \text { student nurses report- } \\
\text { ing significant levels of } \\
\text { affective distress when } \\
\text { screened in the UK }\end{array}$ & $\begin{array}{l}\text { Multimodal stress management; no intervention } \\
\text { control (six 2-hour sessions = } 12 \text { hours) }\end{array}$ & $\begin{array}{l}\text { Derogatis Stress Profile, Beck } \\
\text { \& Srivastava Stress Inventory, } \\
\text { General Health Questionnaire, } \\
\text { STAI, BDI, ways of coping, } \\
\text { absenteeism }\end{array}$ & $\begin{array}{l}\text { Person-di- } \\
\text { rected }\end{array}$ \\
\hline $\begin{array}{l}\text { Lee \& Crockett, } \\
1994(45)\end{array}$ & $\mathrm{RCT}$ & $\begin{array}{l}60 \text { hospital nurses suffer- } \\
\text { ing from insomnia, head- } \\
\text { ache or gastrointestinal } \\
\text { discomfort in Taiwan }\end{array}$ & $\begin{array}{l}\text { Assertiveness training; traditional in-service } \\
\text { program about computer applications in nursing } \\
\text { (six } 2 \text {-hour sessions, } 2 \text { weeks = } 12 \text { hours) }\end{array}$ & $\begin{array}{l}\text { Perceived Stress Scale, Rathus } \\
\text { Assertiveness Schedule }\end{array}$ & $\begin{array}{l}\text { Person-di- } \\
\text { rected }\end{array}$ \\
\hline $\begin{array}{l}\text { Lökk \& Arnetz, } \\
1997(36) ; 2000 \\
(31)^{a}\end{array}$ & $\begin{array}{l}\text { Cluster- } \\
\text { RCT }\end{array}$ & $\begin{array}{l}26 \text { health care personnel } \\
\text { in a geriatric hospital in } \\
\text { Sweden }\end{array}$ & $\begin{array}{l}\text { Support and advice from a psychologist; pas- } \\
\text { sive attendance by psychologist (twenty 1-hour } \\
\text { weekly sessions = } 20 \text { hours) }\end{array}$ & $\begin{array}{l}\text { Blood pressure and pulse rate, } \\
\text { prolactin, cortisol, DHEA, estra- } \\
\text { diol, stress questionnaire }\end{array}$ & $\begin{array}{l}\text { Person-di- } \\
\text { rected }\end{array}$ \\
\hline $\begin{array}{l}\text { McElligott et al, } \\
2003(35)\end{array}$ & $\mathrm{RCT}$ & $\begin{array}{l}20 \text { nurses working at a } \\
\text { tertiary care center in } \\
\text { the US }\end{array}$ & $\begin{array}{l}\text { AMMA therapy; standardized touch therapy pro- } \\
\text { tocol (four } 45 \text {-minute weekly sessions, } 1 \text { month } \\
=3 \text { hours) }\end{array}$ & $\begin{array}{l}\text { Visual analogue scale for anxiety, } \\
\text { blood pressure, heart rate, pulse } \\
\text { oximetry, skin temperature }\end{array}$ & $\begin{array}{l}\text { Person-di- } \\
\text { rected }\end{array}$ \\
\hline $\begin{array}{l}\text { Melchior et al, } \\
1996(37)\end{array}$ & $\mathrm{RCT}$ & $\begin{array}{l}161 \text { psychiatric nurses in } \\
\text { long-stay settings in the } \\
\text { Netherlands }\end{array}$ & $\begin{array}{l}\text { Support and advice about primary nursing } \\
\text { given by nurse managers or quality care coordi- } \\
\text { nators; no intervention control (four months of } \\
\text { preparation = ? hours) }\end{array}$ & Maslach Burnout Inventory & $\begin{array}{l}\text { Organiza- } \\
\text { tional }\end{array}$ \\
\hline $\begin{array}{l}\text { Norvell et al, } \\
1987(40)\end{array}$ & RCT & $\begin{array}{l}12 \text { respiratory therapists } \\
\text { in the US }\end{array}$ & $\begin{array}{l}\text { Stress management program; no intervention } \\
\text { control (eight } 1 \text {-hour weekly sessions, } 2 \text { months } \\
=8 \text { hours) }\end{array}$ & $\begin{array}{l}\text { Maslach Burnout Inventory, } \\
\text { Cohen-Hoberman Inventory of } \\
\text { Physical Symptoms, The Hassles } \\
\text { Scale, The Uplifts Scale }\end{array}$ & $\begin{array}{l}\text { Person-di- } \\
\text { rected }\end{array}$ \\
\hline $\begin{array}{l}\text { Proctor et al, } \\
1998(32)\end{array}$ & $\begin{array}{l}\text { Cluster- } \\
\text { RCT }\end{array}$ & $\begin{array}{l}98 \text { care staff in residential } \\
\text { nursing homes in the UK }\end{array}$ & $\begin{array}{l}\text { Developing knowledge and skills and individ- } \\
\text { ual program planning; no intervention control } \\
\text { (seven 1-hour seminars and weekly visits by a } \\
\text { psychiatric nurse, } 6 \text { months = ? hours) }\end{array}$ & $\begin{array}{l}\text { The Occupational Stress Indicator, } \\
\text { General Health Questionnaire }\end{array}$ & $\begin{array}{l}\text { Organiza- } \\
\text { tional }\end{array}$ \\
\hline $\begin{array}{l}\text { Razavi et al, } 1993 \\
(46)\end{array}$ & RCT & $\begin{array}{l}72 \text { oncology nurses in } \\
\text { Belgium and France }\end{array}$ & $\begin{array}{l}\text { 24-hour psychological training program; no in- } \\
\text { tervention control (eight } 3 \text {-hour weekly sessions, } \\
2 \text { months = } 24 \text { hours) }\end{array}$ & The Nursing Stress Scale & $\begin{array}{l}\text { Organiza- } \\
\text { tional }\end{array}$ \\
\hline Rowe, 1999 (50) & $\mathrm{RCT}$ & $\begin{array}{l}126 \text { health care profes- } \\
\text { sionals from the US }\end{array}$ & $\begin{array}{l}\text { Stress management/adaptive coping training, } \\
\text { with refresher sessions; no intervention control } \\
\text { (six } 1.5 \text {-hour weekly sessions, } 6 \text { weeks = } 9 \\
\text { hours) }\end{array}$ & Maslach Burnout Inventory & $\begin{array}{l}\text { Person-di- } \\
\text { rected }\end{array}$ \\
\hline $\begin{array}{l}\text { Schrijne-maekers } \\
\text { et al, } 2003(33)\end{array}$ & $\begin{array}{l}\text { Cluster- } \\
\text { RCT }\end{array}$ & $\begin{array}{l}300 \text { professional caregiv- } \\
\text { ers in homes for elderly } \\
\text { persons in the Netherlands }\end{array}$ & $\begin{array}{l}\text { Emotion-oriented care training, clinical lessons } \\
\text { and supervision meetings; no intervention con- } \\
\text { trol (two } 1 \text {-hour lessons, } 6 \text {-day training course } \\
\text { and } 3 \text { half-day meetings, } 10 \text { weeks }=50 \text { hours) }\end{array}$ & $\begin{array}{l}\text { Maslach Burnout Inventory, job } \\
\text { satisfaction }\end{array}$ & $\begin{array}{l}\text { Organiza- } \\
\text { tional }\end{array}$ \\
\hline $\begin{array}{l}\text { Tsai \& Crockett, } \\
1993(44)\end{array}$ & RCT & 137 nurses in Taiwan & $\begin{array}{l}\text { Training about stress at work, relaxation, breath- } \\
\text { ing, imagery and meditation; traditional in-ser- } \\
\text { vice education about theory analysis (three 1.5- } \\
\text { hour sessions, } 5 \text { weeks = } 4.5 \text { hours) }\end{array}$ & $\begin{array}{l}\text { Nurse Stress Checklist, Chinese } \\
\text { General Health Questionnaire }\end{array}$ & $\begin{array}{l}\text { Person-di- } \\
\text { rected }\end{array}$ \\
\hline $\begin{array}{l}\text { von Baeyer \& } \\
\text { Krause, } 1983 \text { (47) }\end{array}$ & $\begin{array}{l}\text { Cross- } \\
\text { over }\end{array}$ & $\begin{array}{l}14 \text { nurses in a burn treat- } \\
\text { ment unit in Canada }\end{array}$ & $\begin{array}{l}\text { Cognitive-behavioral stress management train- } \\
\text { ing; no intervention control ( } 3 \text { hours of training } \\
\text { over a week = } 3 \text { hours) }\end{array}$ & STAI & $\begin{array}{l}\text { Person-di- } \\
\text { rected }\end{array}$ \\
\hline $\begin{array}{l}\text { West et al, } 1984 \\
\text { (48) }\end{array}$ & RCT & $\begin{array}{l}60 \text { acute-care hospital } \\
\text { nurses in the US }\end{array}$ & $\begin{array}{l}\text { Stress inoculation training; no intervention con- } \\
\text { trol ( } 1 \text { hour twice a week, } 4 \text { weeks = } 8 \text { hours) }\end{array}$ & $\begin{array}{l}\text { Maslach Burnout Inventory, } \\
\text { job-related tension, life satisfac- } \\
\text { tion STAI, Rathus Assertiveness } \\
\text { Schedule, systolic and diastolic } \\
\text { blood pressure }\end{array}$ & $\begin{array}{l}\text { Person-di- } \\
\text { rected }\end{array}$ \\
\hline $\begin{array}{l}\text { Yung et al, } 2004 \\
(30)\end{array}$ & $\mathrm{RCT}$ & $\begin{array}{l}65 \text { nurse managers in } \\
\text { China }\end{array}$ & $\begin{array}{l}\text { Cognitive relaxation; stretch-release relaxation; } \\
\text { no intervention control (four } 20 \text {-minute weekly } \\
\text { sessions }=1.3 \text { hours) }\end{array}$ & $\begin{array}{l}\text { Chinese-STAI, Chinese General } \\
\text { Health Questionnaire }\end{array}$ & $\begin{array}{l}\text { Person-di- } \\
\text { rected }\end{array}$ \\
\hline
\end{tabular}

a One of the studies was reported in two different articles. 
51). A total of 13 studies used person-directed interventions, including cognitive-behavioral training $(45,47$, $50)$, relaxation training $(30,44)$, music-making (34), therapeutic massage (35), and multicomponent intervention $(31,36,38,40,42,49)$. One study employed a person-work interface intervention consisting of mobilizing support from colleagues and learning participatory problem solving and decision-making skills (39). In the remaining five studies, the intervention [consisting of psychological training programs to improve attitudes, communication skills, and occupational stress $(43,46)$ or changes in work organization, knowledge, skills training, and support and advice from supervisors (32, $33,37)$ ] was directed towards improving the employees' functioning in worktasks.

\section{Results}

\section{Stress}

Person-directed interventions. Two studies $(42,45)$ measured stress directly and reported usable outcome data. Since both used different scales [ie, the Beck and Srivastava Stress Inventory (52) and the perceived stress scale (53)], we used standardized mean differences in the analysis (figure 2). Person-directed interventions reduced stress significantly when compared with no intervention and when measured with these two scales (SMD -0.85, 95\% CI -1.21--0.49). In one study (45) stress remained lower for a month (MD -6.10, 95\% CI -8.44--3.76).

Organizational interventions. The results of one study (43) showed that psychological training (eg, about attitudes and communication skills) reduced stress (mean difference $-0.34,95 \%$ CI -0.62--0.06) when compared with no intervention and when measured with the nursing stress scale (54). This difference became nonsignificant after 6 months (MD -0.19, 95\% CI -0.49-0.11).

\section{Burnout}

Person-directed interventions. Altogether three studies $(38,49,50)$ used the Maslach Burnout Inventory
(MBI) (55). The summary effect of two subscales of the MBI favored the intervention, emotional exhaustion (WMD -5.82, 95\% CI -11.02--0.63) and lack of personal accomplishment (WMD -4.89, 95\% CI -8.71--1.07). There was considerable heterogeneity between the three studies in the meta-analysis (figure 3 ).

The results of one study (50) showed that, when compared with 6 weeks of cognitive-behavioral training, having refresher sessions at 5,11, and 17 months led to significantly lower emotional exhaustion (MD -6.00, 95\% CI -8.16--3.84) and lack of personal accomplishment (MD -5.82, 95\% CI -7.89--3.75) after 2 years.

Table 3. Study quality measured with the Downs \& Black checklist (28). ${ }^{a}$

\begin{tabular}{|c|c|c|c|c|c|}
\hline Study & $\begin{array}{l}\text { Report- } \\
\text { ing (max- } \\
\text { imum 10) }\end{array}$ & $\begin{array}{l}\text { Extern- } \\
\text { al vali- } \\
\text { dity } \\
\text { (maxi- } \\
\text { mum 2) }\end{array}$ & $\begin{array}{c}\text { Intern- } \\
\text { al vali- } \\
\text { dity } \\
\text { (maxi- } \\
\text { mum } \\
13 \text { ) }\end{array}$ & $\begin{array}{c}\text { Power } \\
\text { (max- } \\
\text { imum } \\
5 \text { ) }\end{array}$ & $\begin{array}{c}\text { Total } \\
\text { (max- } \\
\text { imum } \\
30)\end{array}$ \\
\hline \multicolumn{6}{|l|}{ Person-directed interventions } \\
\hline Bittman, et al 2003 (34) & 8 & 2 & 8 & 4 & 22 \\
\hline Cohen-Katz et al, 2005 (49) & 3 & - & 8 & 1 & 12 \\
\hline Ewers et al, 2002 (38) & 8 & 1 & 9 & - & 18 \\
\hline Jones \& Johnston, 2000 (42) & 7 & - & 8 & 3 & 18 \\
\hline Lee \& Crockett, 1994 (45) & 8 & - & 8 & 3 & 19 \\
\hline $\begin{array}{l}\text { Lökk \& Arnetz, } 1997 \text { (36); } \\
2000 \text { (31) }\end{array}$ & 7 & 1 & 8 & 1 & 17 \\
\hline McElligott et al, 2003 (35) & 5 & - & 6 & - & 11 \\
\hline Norvell et al, 1987 (40) & 3 & - & 6 & - & 9 \\
\hline Rowe, 1999 (50) & 8 & - & 9 & 3 & 20 \\
\hline Tsai \& Crockett, 1993 (44) & 5 & 1 & 6 & 4 & 16 \\
\hline $\begin{array}{l}\text { von Baeyer \& Krause, } \\
1983 \text { (47) }\end{array}$ & 8 & 1 & 9 & - & 18 \\
\hline West et al, 1984 (48) & 6 & - & 8 & 1 & 15 \\
\hline Yung et al, 2004 (30) & 10 & - & 9 & 2 & 21 \\
\hline \multicolumn{6}{|c|}{ Person-work interface interventions } \\
\hline Heaney et al, 1995 (39) & 5 & - & 6 & 5 & 16 \\
\hline \multicolumn{6}{|l|}{ Organizational interventions } \\
\hline Delvaux et al, 2004 (43) & 8 & 1 & 10 & 4 & 23 \\
\hline Melchior et al, 1996 (37) & 7 & 1 & 7 & 4 & 19 \\
\hline Proctor et al, 1998 (32) & 6 & - & 7 & 3 & 16 \\
\hline Razavi et al, 1993 (46) & 7 & - & 8 & 3 & 18 \\
\hline $\begin{array}{l}\text { Schrijnemaekers et al, } \\
2003 \text { (33) }\end{array}$ & 7 & 1 & 10 & 5 & 23 \\
\hline
\end{tabular}

a Studies that scored $75 \%$ or more (10 points) on internal validity were considered high quality.

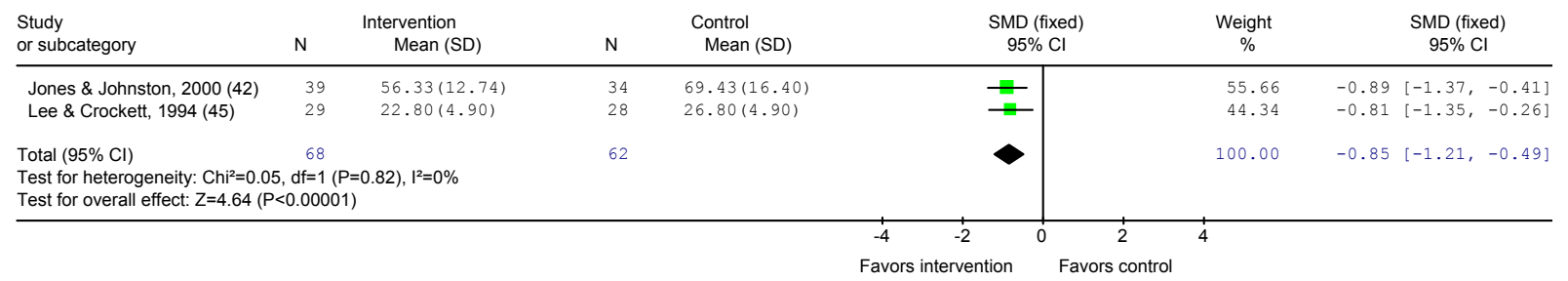

Figure 2. Person-directed intervention versus no intervention measured with stress scales. 


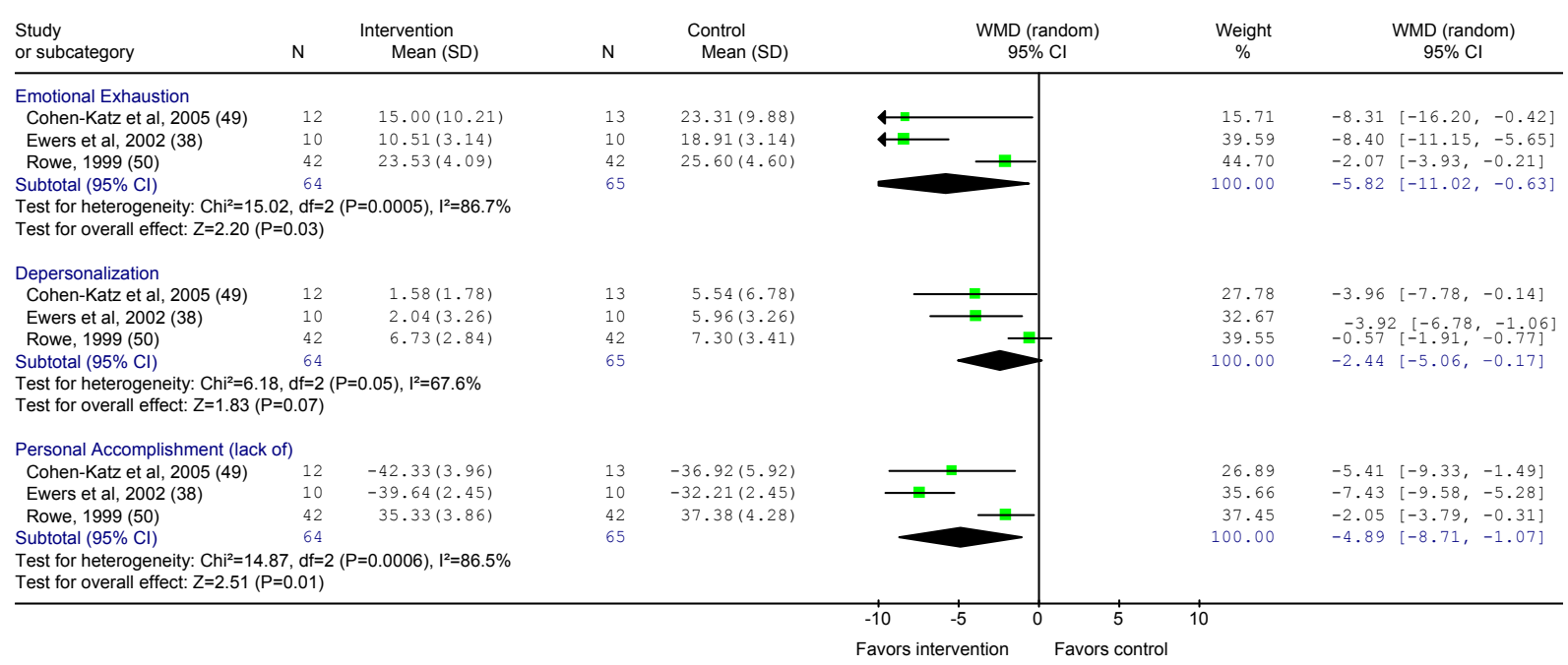

Figure 3. Person-directed intervention versus no intervention measured with the Maslach Burnout Inventory.

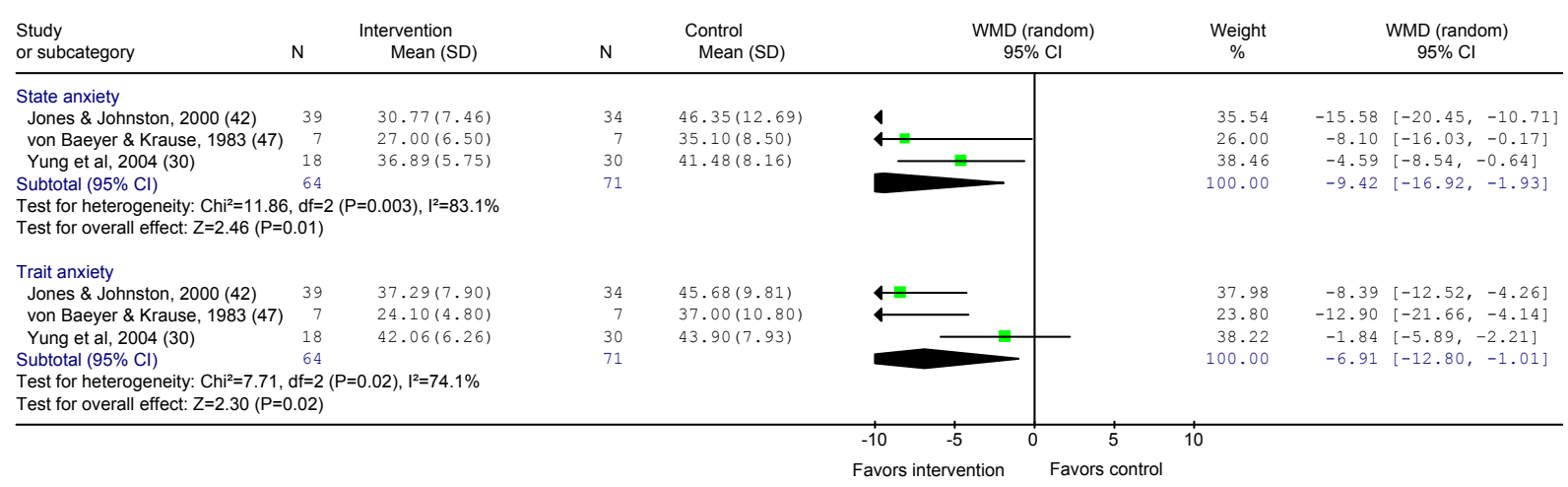

Figure 4. Person-directed intervention versus no intervention measured with the State Trait Anxiety Inventory.

Organizational interventions. According to one study (37), support and advice given by nurse managers or quality-care coordinators reduced symptoms on one of the subscales of the MBI: depersonalization (MD $-1.14,95 \%$ CI $-2.18--0.10$ ) when compared with no intervention.

\section{Anxiety}

Person-directed interventions. According to three studies $(30,42,47)$, person-directed interventions significantly reduced both state anxiety (WMD -9.42 , 95\% CI $-16.92-1.93$ ) and trait anxiety (WMD -6.91 , $95 \%$ CI $-12.80--1.01)$ when compared with no intervention, as measured with the state-trait anxiety inventory (56). There was considerable heterogeneity, one study (30) showing lower decreases on both scales than the other two studies (figure 4). According to two studies $(30,42)$, the state anxiety (WMD -8.31 , 95\% CI $-11.49--5.13$ ) and trait anxiety (WMD -4.09 , $95 \%$ CI $-7.60--0.58$ ) scores remained lower in the intervention group for at least 1 month.

\section{General symptoms}

Person-directed interventions. According to two studies $(30,42)$, person-directed interventions did not reduce general symptoms significantly more than no intervention (WMD -11.87, 95\% CI -27.24-3.49) when measured with the General Health Questionnaire (57). The results of one study (30) showed that cognitive relaxation training maintained the reduction in scores of the General Health Questionnaire for at least 1 month, whereas stretch-release relaxation training did not (MD $-7.10,95 \%$ CI $-10.58--3.62)$.

Organizational interventions. One study (32) showed that a combination of training knowledge and skills and individual program-planning decreased general symptoms (MD -2.90, 95\% CI -5.16--0.64) when compared with no intervention and when measured with the General Health Questionnaire (57).

\section{Levels of evidence}

The qualitative analyses agreed with the results of the meta-analyses. [See table 4 for the results of our grading 
Table 4. Results of the studies unfit for meta-analysis according to outcome.

\begin{tabular}{|c|c|c|c|c|c|c|}
\hline Outcome & $\begin{array}{l}\text { High-qual- } \\
\text { ity positive } \\
\text { effect (strong } \\
\text { evidence) }\end{array}$ & $\begin{array}{l}\text { Low-quality positive effect (limited } \\
\text { evidence) }\end{array}$ & $\begin{array}{l}\text { High-qual- } \\
\text { ity negative } \\
\text { effect (strong } \\
\text { evidence) }\end{array}$ & $\begin{array}{l}\text { Low -qual- } \\
\text { ity negative } \\
\text { effect (limited } \\
\text { evidence) }\end{array}$ & $\begin{array}{l}\text { High-quality } \\
\text { inconsistent } \\
\text { effect }\end{array}$ & $\begin{array}{l}\text { Low-quality inconsistent } \\
\text { effect }\end{array}$ \\
\hline \multicolumn{7}{|c|}{ Person-directed interventions } \\
\hline Stress & $\cdot$ & $\begin{array}{l}\text { Jones \& Johnston, } 2000 \text { (42); } \\
\text { Lee \& Crockett, } 1994 \text { (45); Tsai } \\
\text { \& Crockett, } 1993 \text { (44); West et al, } \\
1984 \text { (48) }\end{array}$ & . & . & . & Rowe, 1999 (50) \\
\hline \multirow[t]{2}{*}{$\begin{array}{l}\text { Emotional } \\
\text { exhaustion }\end{array}$} & . & $\begin{array}{l}\text { Cohen-Katz et al, } 2005 \text { (49); Ewers } \\
\text { et al, } 2002 \text { (38); Norvell et al, } 1987 \\
\text { (40); Rowe, 1999 (50); West et al, } \\
1984 \text { (48) }\end{array}$ & . & . & . & Bittman et al, 2003 (34) \\
\hline & . & $\begin{array}{l}\text { Ewers et al, } 2002 \text { (38); Rowe, } 1999 \\
(50)\end{array}$ & . & . & . & $\begin{array}{l}\text { Bittman et al, } 2003 \text { (34); } \\
\text { Cohen-Katz et al, } 2005 \text { (49); } \\
\text { Norvell et al, } 1987 \text { (40); West } \\
\text { et al, } 1984 \text { (48) }\end{array}$ \\
\hline $\begin{array}{l}\text { Personal } \\
\text { accomplishment }\end{array}$ & $\cdot$ & $\begin{array}{l}\text { Cohen-Katz et al, } 2005 \text { (49); Ewers } \\
\text { et al, } 2002 \text { (38); Rowe, } 1999 \text { (50); } \\
\text { West et al, } 1984 \text { (48) }\end{array}$ & . & . & . & $\begin{array}{l}\text { Bittman et al, } 2003(34) \\
\text { Norvell et al, } 1987 \text { (40) }\end{array}$ \\
\hline State anxiety & . & $\begin{array}{l}\text { Jones \& Johnston, } 2000 \text { (42); von } \\
\text { Baeyer \& Krause, } 1983 \text { (47): West } \\
\text { et al, } 1984 \text { (48); Yung et al, } 2004 \\
\text { (30) }\end{array}$ & . & . & . & . \\
\hline Trait anxiety & . & $\begin{array}{l}\text { Jones \& Johnston, } 2000 \text { (42); von } \\
\text { Baeyer \& Krause, } 1983 \text { (47); West } \\
\text { et al, } 1984 \text { (48) }\end{array}$ & . & · & · & Yung 2004 (30) \\
\hline $\begin{array}{l}\text { General } \\
\text { symptoms }\end{array}$ & . & $\begin{array}{l}\text { Jones \& Johnston, } 2000 \text { (42); Yung } \\
\text { et al, } 2004 \text { (30) }\end{array}$ & $\cdot$ & . & . & $\begin{array}{l}\text { Cohen-Katz et al, } 2005 \text { (49); } \\
\text { Norvell et al, } 1987 \text { (40); Tsai } \\
\text { \& Crockett, } 1993 \text { (44) }\end{array}$ \\
\hline \multicolumn{7}{|c|}{ Person-work interface interventions } \\
\hline $\begin{array}{l}\text { General } \\
\text { symptoms }\end{array}$ & $\cdot$ & . & . & . & . & Heaney et al, 1995 (39) \\
\hline \multicolumn{7}{|c|}{ Organizational interventions } \\
\hline Stress & $\begin{array}{l}\text { Delvaux et al, } \\
2004(43)\end{array}$ & . & . & . & . & Proctor et al, 1998 (32) \\
\hline $\begin{array}{l}\text { Emotional } \\
\text { exhaustion }\end{array}$ & . & . & . & . & & Melchior et al, 1996 (37) \\
\hline & . & . & . & . & & Melchior et al, 1996 (37) \\
\hline $\begin{array}{l}\text { Personal } \\
\text { accomplishment }\end{array}$ & & . & . & . & . & Melchior et al, 1996 (37) \\
\hline $\begin{array}{l}\text { General } \\
\text { symptoms }\end{array}$ & . & Proctor et al, 1998 (32) & . & . & . & . \\
\hline
\end{tabular}

of the evidence and table 1 for our criteria for judging what was considered strong, limited, inconsistent, or no evidence.] Studies that scored $75 \%$ or more (10 points) on internal validity on the Downs \& Black checklist (28) were considered high in quality.

\section{Discussion}

From the meta-analyses and qualitative analyses, we can conclude that there is limited evidence that persondirected interventions among health care workers effectively reduce the levels of burnout, anxiety, and stress and that organizational interventions reduce the levels of stress, burnout, and general symptoms.

We could not subdivide the person-directed interventions further because almost all of the studies used several different components. Most of the studies claimed that it is possible to change the participants' cognitions about stressful elements at work. We refrained from further sensitivity analyses based on differences (i) in quality because most of the studies scored about mid-range on the quality scale and (ii) in the content of the interventions because most of the interventions were complex. It is interesting to note that the studies involving organizational interventions scored higher on the quality checklist even though it is more difficult to carry out interventions targeted at worktasks (21). This finding is surprising because the implementation of organizational interventions is dependent on more stakeholders than the patient alone and these types of interventions are therefore more difficult to organize and keep under control. The authors of the included organizational intervention studies not only succeeded in randomizing workplaces, they did it well. One would have not only expected these studies to have weaker study designs as is usually the case (21), but also to display the largest effects since 
they aimed higher in the hierarchy of controls. [See the report of Lamontagne et al (58).] Since there were no direct comparisons, we did not attempt to compare the outcomes of person-directed with person-work interface or organizational interventions, as indirect comparisons are susceptible to bias (59). However, it is clear that the problem of attrition is bigger in the latter, as the personal interests of participants apparently differ in this type of intervention. All of the studies included in this review used standardized and previously validated self-report scales to measure the outcomes [eg, Maslach Burnout Inventory (55), General Health Questionnaire (57), State Trait Anxiety Inventory (56), and Beck \& Srivastava Stress Inventory (52)].

\section{Limitations of the review}

In the meta-analyses of person-directed interventions there was considerable heterogeneity, two studies (30, 50) showing less decrease in burnout and anxiety immediately after the intervention. However, in the studies in which follow-up lasted at least a month, these differences disappeared. We could not find a good explanation for the heterogeneity in the results of the studies with post-intervention measurements only. The quality of the evidence that we found was not very high. Some of the studies applied rigorous methods but contended with attrition problems. Most of the randomized controlled trials were small, and, in all but one (42) of the included studies, the method of randomization was not reported or not valid. It was also difficult to get a good impression of the concealment of allocation to the researchers. We assumed that outcomes that were measured by a questionnaire were reported blind to the researchers. Even though we found significant results, it is difficult to say how the results are related to the clinical relevance of the changes achieved. With the Maslach Burnout Inventory (55), there is no generally accepted change that would be regarded as clinically relevant (Wilmar Schaufeli, personal communication). Since most of the studies had only a small sample size and all of them reported positive outcomes, it is conceivable that there may have been publication bias. However, the extent of publication bias is impossible to assess, as all of the studies did not report outcomes that could be used for a statistical analysis.

\section{Comparison with other reviews}

This review used more rigorous inclusion criteria and found more and better-quality evidence than previous reviews about stress interventions directed towards health care workers $(7,26)$. Another review involved a metaanalysis of interventions to prevent or treat stress in all occupations (25). The review synthesized all of the study outcomes available in primary studies. This approach makes it difficult to decipher the meaning of their findings. In contrast to our review, they concluded that there was no evidence for work-directed or person-work interface interventions (25). Due to the different samples of studies (our review contains 13 studies that theirs does not) and methods used, it is difficult to explain this contrasting finding. A recent review about interventions to improve the morale of staff was restricted to mental health care workers only (60).

\section{Implications}

The results of this review show that stress management interventions can lead to positive health effects among health care personnel. There is evidence from one trial (30) that interventions that contain cognitive elements yield better results than those with behavioral elements. However, before large-scale implementation can be advised, larger and better-quality trials are needed. It would also be good to know what the current prevalence of various stress management strategies is. According to our own experience, it is much more common to measure various indices of stress than it is to do something about it. None of the studies looked specifically at stress reduction among physicians, probably because this professional group is often more reluctant to participate. Since physicians usually have more decision latitude or control, autonomy, possibilities for development, and rewards than nurses do, it is logical to assume that they may also need especially tailored stress management interventions. It is therefore difficult to say whether our results can be generalized also to physicians. Studies are needed that contrast various stress- or burnout-reducing techniques with one another. Studies that contrast organizational and person-work interface interventions with person-directed interventions will show whether or not one type of intervention is more effective in reducing stress levels than the other.

\section{Acknowledgments}

This study was conducted as a Cochrane systematic review under the auspices of the Cochrane Depression, Anxiety and Neurosis Group. In addition, we would like to thank the Cochrane Occupational Health Field and all the authors that provided additional information regarding their studies.

The Finnish Ministry of Social Affairs and Health enabled the finalization of this review. The Ministry did not have any role in the design or conduct of the study; the collection, management, analysis, or interpretation of the data; or the preparation, review, or approval of the manuscript. 
This systematic review was prepared under the aegis of The Cochrane Collaboration, an international organization that aims to help people make well-informed decisions about health care by preparing, maintaining, and promoting the accessibility of systematic reviews of the effects of health care interventions. The Collaboration's publication policy permits journals to publish reviews but also permits The Cochrane Collaboration also to publish and disseminate such reviews.

\section{References}

1. Karasek R. The stress-disequilibrium theory: chronic disease development, low social control, and physiological de-regulation. Med Lav. 2006;97:258-71.

2. Siegrist J. Adverse health effects of high-effort/low-reward conditions. J Occup Health Psychol. 1996;1:27-41.

3. Cooper CL, Dewe P, O'Driscoll MP. Organizational stress. London: Sage; 2001

4. Schaufeli WB, Buunk BP. Burnout: An overview of 25 years of research and theorizing. In: Schabracq MJ, Winnubst JAM, Cooper CL, editors. The handbook of work and health psychology. Chichester (United Kingdom): John Wiley \& Sons, Ltd; 2003:383-424.

5. Bamber MR. CBT for Occupational stress in health professionals: Introducing a schema-focused approach. Hove (United Kingdom): Routledge; 2006.

6. Weinberg A, Creed F. Stress and psychiatric disorder in healthcare professionals and hospital staff. Lancet. 2000;355:533-7.

7. Michie S, Williams S. Reducing work related psychological ill health and sickness absence: a systematic literature review. Occup Environ Med. 2003;60:3-9.

8. Leiter MP, Harvie P, Frizzell C. The correspondence of patient satisfaction and nurse burnout. Soc Sci Med. 1998;47:1611-7.

9. Firth-Cozens J, Greenhalgh J. Doctors' perceptions of the links between stress and lowered clinical care. Soc Sci Med. 1997;44:1017-22.

10. Firth-Cozens J, Payne R, editors. Stress in health professionals. Chichester (United Kingdom): Wiley; 1999.

11. Jacobson BH, Aldana SG, Goetzel RZ, Vardell KD, Adams TB, Piedras RJ. The relationship between perceived stress and self-reported illness-related absenteeism. Am J Health Promot. 1996;11:54-61.

12. Raiger J. Applying a cultural lens to the concept of burnout. J Transcult Nurs. 2005;16:71-6.

13. McNeely E. The consequences of job stress for nurses' health: time for a check-up. Nurs Outlook. 2005;53:291-9.

14. French SE, Lenton R, Walters V, Eyles J. An empirical evaluation of an expanded Nursing Stress Scale. J Nurs Meas. 2000;8:161-78.

15. McVicar A. Workplace stress in nursing: a literature review. J Adv Nurs. 2003;44:633-42.

16. Deckard G, Meterko M, Field D. Physician burnout: an examination of personal, professional, and organizational relationships. Med Care. 1994;32:745-54.

17. Karasek R. Stress prevention through work reorganization: a summary of 19 international case studies. Cond Work Dig. 1992;11:23-42.

18. Maslach C. Burnout: the cost of caring. New York (NY): Prentice-Hall; 1982.
19. Ramirez AJ, Graham J, Richards MA, Cull A, Gregory WM. Mental health of hospital consultants: the effects of stress and satisfaction at work. Lancet. 1996;347:724-8.

20. Embriaco N, Azoulay E, Barrau K, Kentish N, Pochard F, Loundou A, et al. High level of burnout in intensivists: prevalence and associated factors. Am J Respir Crit Care Med. 2007;175:686-92.

21. Semmer NK. Job stress interventions and the organization of work. Scand J Work Environ Health. 2006;32(6, special issue):515-27.

22. DeFrank RS, Cooper CL. Worksite stress management interventions: their effectiveness and conceptualisation. J Managerial Psychol. 1987;2:4-10.

23. Murphy S. Stress management in work settings: a critical review of the health effects. Am J Health Promot. 1995;11:112-35.

24. van der Hek H, Plomp HN. Occupational stress management programmes: a practical overview of published effect studies. Occup Med [London]. 1997;47:133-41.

25. van der Klink JJ, Blonk RW, Schene AH, van Dijk FJ. The benefits of interventions for work-related stress. Am J Public Health. 2001;91:270-6.

26. Mimura $\mathrm{C}$, Griffiths $\mathrm{P}$. The effectiveness of current approaches to workplace stress management in the nursing profession: an evidence based literature review. Occup Environ Med. 2003;60:10-5.

27. Marine A, Ruotsalainen J, Serra C, Verbeek J. Preventing occupational stress in healthcare workers. Cochrane Database Syst Rev. 2006;CD002892.

28. Downs SH, Black N. The feasibility of creating a checklist for the assessment of the methodological quality both of randomised and non-randomised studies of health care interventions. J Epidemiol Community Health. 1998;52:377-84.

29. van Tulder M., Furlan A, Bombardier C, Bouter L. Updated method guidelines for systematic reviews in the cochrane collaboration back review group. Spine. 2003;28:1290-9.

30. Yung PM, Fung MY, Chan TM, Lau BW. Relaxation training methods for nurse managers in Hong Kong: a controlled study. Int J Ment Health Nurs. 2004;13:255-61.

31. Lökk J, Arnetz B. Impact of management change and an intervention program on health care personnel. Psychother Psychosom. 2000;69:79-85.

32. Proctor R, Stratton-Powell H, Tarrier N, Burns A. The impact of training and support on stress among care staff in nursing and residential homes for the elderly. J Ment Health. 1998;7:59-71.

33. Schrijnemaekers VJ, van Rossum E, Candel MJ, Frederiks CM, Derix MM, Sielhorst H, et al. Effects of emotion-oriented care on work-related outcomes of professional caregivers in homes for elderly persons. J Gerontol Soc Sci. 2003;58:S50-7.

34. Bittman B, Bruhn KT, Stevens C, Westengard J, Umbach PO. Recreational music-making: a cost-effective group interdisciplinary strategy for reducing burnout and improving mood states in long-term care workers. Adv Mind Body Med. 2003;19:4-15.

35. McElligott D, Holz MB, Carollo L, Somerville S, Baggett M, Kuzniewski S, et al. A pilot feasibility study of the effects of touch therapy on nurses. J N Y State Nurses Assoc. 2003;34:16-24.

36. Lökk J, Arnetz B. Psychophysiological concomitants of organizational change in health care personnel: effects of a controlled intervention study. Psychother Psychosom. 1997;66:74-77.

37. Melchior ME, Philipsen H, Abu-Saad HH, Halfens RJ, van de Berg AA, Gassman P. The effectiveness of primary nursing on burnout among psychiatric nurses in long-stay settings. J Adv 
Nurs. 1996;24:694-702.

38. Ewers P, Bradshaw T, McGovern J, Ewers B. Does training in psychosocial interventions reduce burnout rates in forensic nurses? J Adv Nurs. 2002;37:470-6.

39. Heaney CA, Price RH, Rafferty J. Increasing coping resources at work: a field experiment to increase social support, improve work team functioning, and enhance employee mental health. J Organ Behav. 1995; 16:335-52.

40. Norvell N, Belles D, Brody S, Freund A. Worksite stress management for medical care personnel: results from a pilot program. J Spec Group Work. 1987;118-26.

41. Proctor R, Stratton-Powell H, Tarrier N, Burns A. The impact of training and support on stress among care staff in nursing and residential homes for the elderly. J Ment Health. 1998;7:59-71.

42. Jones MC, Johnston DW. Evaluating the impact of a worksite stress management programme for distressed students: a randomised controlled trial. Psychol Health. 2000;15:689-706.

43. Delvaux N, Razavi D, Marchal S, Bredart A, Farvacques C, Slachmuylder JL. Effects of a 105 hours psychological training program on attitudes, communication skills and occupational stress in oncology: a randomised study. Br J Cancer. 2004;90:106-14.

44. Tsai SL, Crockett MS. Effects of relaxation training, combining imagery, and meditation on the stress level of Chinese nurses working in modern hospitals in Taiwan. Issues Ment Health Nurs. 1993;14:51-66.

45. Lee S, Crockett MS. Effect of assertiveness training on levels of stress and assertiveness experienced by nurses in Taiwan, Republic of China. Issues Ment Health Nurs. 1994;15:419-32.

46. Razavi D, Delvaux N, Marchal S, Bredart A, Farvacques C, Paesmans M. The effects of a 24-h psychological training program on attitudes, communication skills and occupational stress in oncology: a randomised study. Eur J Cancer. 1993;29A:1858-63.

47. von Baeyer C., Krause L. Effectiveness of stress management training for nurses working in a burn treatment unit. Int J Psychiatry Med. 1983;13:113-26.

48. West DJ, Jr., Horan JJ, Games PA. Component analysis of oc- cupational stress inoculation apllied to registered nurses in an acute care hospital setting. J Couns Psychol. 1984;31:209-18.

49. Cohen-Katz J, Wiley SD, Capuano T, Baker DM, Kimmel $\mathrm{S}$, Shapiro $\mathrm{S}$. The effects of mindfulness-based stress reduction on nurse stress and burnout, part. Holist Nurs Pract. 2005;19:26-35.

50. Rowe MM. Teaching health-care providers coping: results of a two-year study. J Behav Med. 1999;22:511-27.

51. Taris TW, Kompier MAJ, Geurts SAE, Schreurs PJG, Schaufeli WB, De Boer E, et al. Stress management interventions in the Dutch domiciliary care sector: findings from 81 organizations. Int J Stress Manag. 2003;10:297-325.

52. Beck DL, Srivastava R. Perceived level and sources of stress in baccalaureate nursing students. J Nurs Educ. 1991;30:127-33.

53. Cohen S, Kamarck T, Mermelstein R. A global measure of perceived stress. J Health Soc Behav. 1983;24:385-96.

54. Gray-Toft P, Anderson JG. The nursing stress scale: development of an instrument. J Psychopathol Behav Assess. 1981;3:11-23.

55. Maslach C, Jackson SE, Leiter SP. Maslach burnout inventory manual. 3rd ed. Palo Alto (CA): Consulting Psychologists Press; 1996.

56. Spielberger CD, Gorsuch RL, Lushene RE. Manual for the state-trait anxiety inventory (self-evaluation questionnaire). Palo Alto (CA): Mind Garden; 1970.

57. Goldberg D, Williams P. A user's guide to the general health questionnaire. London: NFER-Nelson; 1991.

58. LaMontagne AD, Keegel T, Louie AM, Ostry A, Landsbergis PA. A systematic review of the job-stress intervention evaluation literature, 1990-2005. Int J Occup Environ Health. 2007;13:268-80.

59. Glenny AM, Altman DG, Song F, Sakarovitch C, Deeks JJ, D'Amico R, et al. Indirect comparisons of competing interventions. Health Technol Assess. 2005;9:iii-iv.

60. Gilbody S, Cahill J, Barkham M, Richards D, Bee P, Glanville J. Can we improve the morale of staff working in psychiatric units?: a systematic review. J Ment Health. 2006;15:7-17.

Received for publication: 10 September 2007

\section{Appendix}

\section{MEDLINE search strategy}

[randomised controlled trial.pt. OR controlled clinical trial.pt. OR randomised controlled trials OR random allocation OR double blind method OR single blind method OR clinical trial.pt. OR exp Clinical trial OR (clin\$ adj25 trial\$). ti,ab. OR ((singl\$ OR doubl\$ OR trebl\$ OR tripl\$) adj25 (blind \$ OR mask\$)).ti,ab. OR placebos OR placebos.ti,ab. OR random.ti,ab. OR research design OR comparative study OR exp "Evaluation and Follow Up" OR follow up studies OR prospective studies OR (control\$ OR prospectiv\$ OR volunteer\$).ti,ab.] NOT (animal NOT human)

AND

exp Burnout, professional OR burnout.tw. OR exp job satisfaction OR job satisfaction.tw. OR (mental adj25 health adj25 professional\$).ab,ti. OR (stress adj25 professional\$).ab,ti. OR (stress adj25 occupational).ab,ti. OR exp anxiety OR exp anxiety disorders OR anxiety.ti. OR exp Depression OR depression.ti.

AND

(exp Health personnel OR (health.ti,ab. AND personnel.ti,ab.) OR Nursing OR nurse.ti,ab. OR nursing.ti,ab)) AND LIMIT human 